\title{
Toward a Better Guidance in Wearable Electronic Orientation Aids
}

\author{
Slim Kammoun, Marc J.-M. Macé, Bernard Oriola, and Christophe Jouffrais \\ IRIT, CNRS \& University of Toulouse, \\ Toulouse, France \\ \{Kammoun, Mace, Oriola, Jouffrais\} @irit.fr
}

\begin{abstract}
Electronic Orientation Aids (EOA) usually guide visually impaired pedestrians using turn-by-turn instructions. However, several studies have demonstrated that mental representations of space seem difficult to build when turn-by-turn instructions only are provided. This is a crucial point as getting an accurate spatial representation of the surroundings can make the difference between successful and unsuccessful navigation. In this paper, we describe components of a guidance system designed to provide a better service in wearable electronic orientation aids for the visually impaired. They especially include non-visual landmarks and points of interest that are used as environmental features to improve mental spatial representations.
\end{abstract}

Keywords: Visually impaired, Electronic Orientation Aids, navigation, orientation, mobility, assistive technology, guidance.

\section{Introduction}

In assisted navigation for the pedestrian, guidance instructions usually rely on turnby-turn instructions (e.g. "turn left in fifty meters"). For visually impaired as well as sighted pedestrians, landmarks play an important role in decision-making when traveling. They are used either as reorientation or confirmation points, and are significant elements in the communication of route directions, which shows that they are useful to elaborate mental representations of space. Hence providing the visually impaired with these environmental features during the guidance process is critical for the usability of EOAs, and therefore for successful navigation tasks, with the parallel benefit of contributing to the elaboration of mental representation of space.

In this paper we defined primary steps toward a better guidance process by taking into account the needs of the visually impaired and the potential of technologies such as wearable sensors, Global Positioning System (GPS), and Geographic Information System (GIS). Guidance consists first in identifying the location of the VI user relative to the expected trajectory, and then in providing him/her with appropriate direction instructions and pertinent information about the surroundings. Hence we defined guidance as three main steps: 1) Route selection to compute the optimal itinerary, 2) Tracking to estimate the location of the traveler, 3) Display of navigation instructions and selected spatial cues to guide the traveler and improve his mental representation of the environment. 


\section{Guidance in Electronic Orientation Aids}

In the following section, we decompose and describe the guidance process generally used in EOAs, with a particular focus on the differences we propose compared to existing systems.

\subsection{GIS and Route Selection}

Route selection is defined as the procedure of choosing an optimal path between origin and destination. Based on multiple brainstorming and interviews with VI people and orientation and mobility (O\&M) instructors, Kammoun et al. [1] proposed an annotation of geographical data that takes into account the needs of VI pedestrians. This classification included three main classes: 1) The pedestrian network (sidewalks, pedestrian crossing, etc.); 2) Non-visual landmarks (decision or confirmation points) corresponding to locations that can be detected by the VI pedestrian (e.g. pavement texture or inclination, street furniture, specific odor/sound, etc.); 3) Points of Interest (POIs) such as places or objects that are potential destinations (e.g. public buildings, shops, etc.). Landmarks and POIs are also useful clues to construct a more complete mental representation of the environment while traveling. Using such annotations, it is possible to select the route most adapted to the needs of VI pedestrians [1].

\subsection{Tracking User Location}

Geolocation based on GPS is the most common technique used in EOAs. However, positioning precision with GPS alone is rarely better than 10 to 20 meters in many environments, mostly in cities where urban canyons prevent direct line of sight with the satellites. Several approaches have been developed to improve GPS precision. These solutions include dead-reckoning algorithms, differential GPS (D-GPS) or electronic location identifiers, such as RFID tags, WLAN networks or Bluetooth beacons which are widely used in indoor environments. In order to get a better pedestrian positioning, it is possible to fuse vision-based (local) and GPS-based (global) positioning signals. The EOA described in the following sections included a GPS and embedded stereo cameras. An ultra-rapid recognition and localization algorithm detects geolocated visual landmarks in the environment. The location of the pedestrian relative to the geolocated visual landmarks is then used to refine the global position of the pedestrian on the digitized map.

\subsection{Rendering of Descriptive and Spatial Information}

Interviews of VI users and O\&M instructors made clear that EOAs should provide both directional instructions and information about the surroundings. To display turnby-turn instructions and space-related information to the VI traveler, an adapted interface is required, which must rely on non-visual (e.g. auditory or somatosensory) modalities. Evaluation showed that both TTS (see e.g. [2] [3]) and spatialized sounds [4] may provide efficient rendering. While TTS technique is perfectly adapted to turnby-turn instruction, binaural synthesis can be used to generate sounds at any given spatial location relative to the listener. Combination of TTS and binaural synthesis is 
an interesting solution to display both guidance instructions and space configuration information. Another interesting wearable device consists of actuators mounted on a waist belt [5], but it provides information regarding heading direction only.

\section{The NAVIG Orientation Aid}

The NAVIG system (Navigation Assisted by artificial VIsion and GNSS) is an assistive device whose objective is to increase the autonomy of visually impaired users in known and unknown environments [6]. Here, we focus on the guidance process that we have implemented in the prototype. Positioning data was provided by a GPS receiver combined with a wearable inertial measurement unit (IMU) and stereo cameras [7]. The IMU included accelerometers, an electronic compass and a pedometer and was developed by the firm and project partner Navocap. The stereo cameras allowed to estimate the traveler's location (direction and distance) relative to environmental features that were geolocated and tagged in the GIS database. This vision-based localization module was developed by the firm and project partner SpikeNet. The GIS module consisted in an adapted digitized map where walking areas (e.g. sidewalks, pedestrian crossings, etc.) and environmental features (visual points for the cameras as well as non-visual landmarks and points of interest for the user) were added to additional layers. Interaction with the user was based on speech recognition. For output interaction, we used a real-time binaural synthesis module (designed by the project partner LIMSI) to render directions, and a TTS module to describe surrounding landmarks and POIs.

When a route was selected between two points, several sections defined by two successive geolocalized Itinerary Points (IPs) were generated. Each section also contained a list of geolocalized landmarks and POIs extracted from the GIS. Using IPs, the system then generated turn-by-turn instructions based on the traveler position and direction provided by the positioning component. Both landmarks and POIs were displayed to provide the user with information about her/his local environment. To track the user location, we used a simple algorithm based on activation fields. To trigger the display of information, a radius was defined according to each type of point. The radiuses of the different activation fields were determined during preliminary tests that were conducted with the positioning module. TTS and Spatialized TTS were used to display environmental information when reaching activation fields.

\section{Conclusion and Future Work}

In this paper, we focused on the general guidance process designed for an Electronic Orientation Aid for the visually impaired. This process was decomposed in three important components: (1) route selection procedure to compute the optimal itinerary, (2) user tracking to estimate the location of the traveler, and (3) improvement of the mental representation of space through the display of adapted instructions and selected surrounding elements. We implemented this function in a prototype of the NAVIG system, a wearable orientation aid designed to overcome blind mobility 
difficulties. To be effective, this guidance process relies on an adapted GIS, an improved positioning module (compared to GPS alone), and an adapted user interface based on spatialized sounds and TTS. Further work is necessary to evaluate the impact of this guidance process on navigation tasks. In addition, as mental representations of space are a key factor in the successful accomplishment of navigation tasks, it will also be of interest to evaluate the quality of mental representation built through this guidance process.

Acknowledgments. This work was supported by the French National Research Agency (ANR) through TecSan program (project NAVIG ANR-08TECS-011) and the Midi-Pyrénées region through APRRTT program. The NAVIG consortium includes three research labs (IRIT, LIMSI, and CerCo), two companies (SpikeNet Technology, and NAVOCAP), the Institute for Young Blind of Toulouse (CESDVIJA), and the community of Grand Toulouse.

\section{References}

1. Kammoun, S., Dramas, F., Oriola, B., Jouffrais, C.: Route selection algorithm for Blind pedestrian. In: Proceedings of the International Conference on Control Automation and Systems (ICCAS), pp. 2223-2228. IEEE, Los Alamitos (2010)

2. Gaunet, F.: Verbal guidance rules for a localized wayfinding aid intended for blind pedestrians in urban areas. Universal Access in the Information Society 4, 338-353 (2006)

3. Golledge, R., Marston, J., Costanzo, C.M.: Assistive Devices and Services for the Disabled: Auditory Signage and the Accessible City for Blind or Vision-Impaired Travelers. Signs (1998)

4. Loomis, J.M., Golledge, R.G., Klatzky, R.L.: Navigation System for the Blind: Auditory Display Modes and Guidance. Presence: Teleoperators and Virtual Environments 7, $193-$ 203 (1998)

5. Heuten, W., Henze, N., Boll, S., Pielot, M.: Tactile wayfinder: a non-visual support system for wayfinding. In: Proceedings of the 5th Nordic Conference on Human-computer Interaction: Building Bridges, pp. 172-181. ACM, New York (2008)

6. Katz, B., Truillet, P., Thorpe, S., Jouffrais, C.: NAVIG: Navigation Assisted by Artificial Vision and GNSS. In: Workshop on Multimodal Location Based Techniques for Extreme Navigation, Finland, pp. 1-4 (2010)

7. Brilhault, A., Kammoun, S., Gutierrez, O., Truillet, P., Jouffrais, C.: Fusion of Artificial Vision and GPS to Improve Blind Pedestrian Positioning. In: Proceedings of the IFIP International Conference on Conference on New Technologies, Mobility and Security (NTMS), pp. 1-5 (2011) 